\title{
MathML / XML series: What is MathML?
}

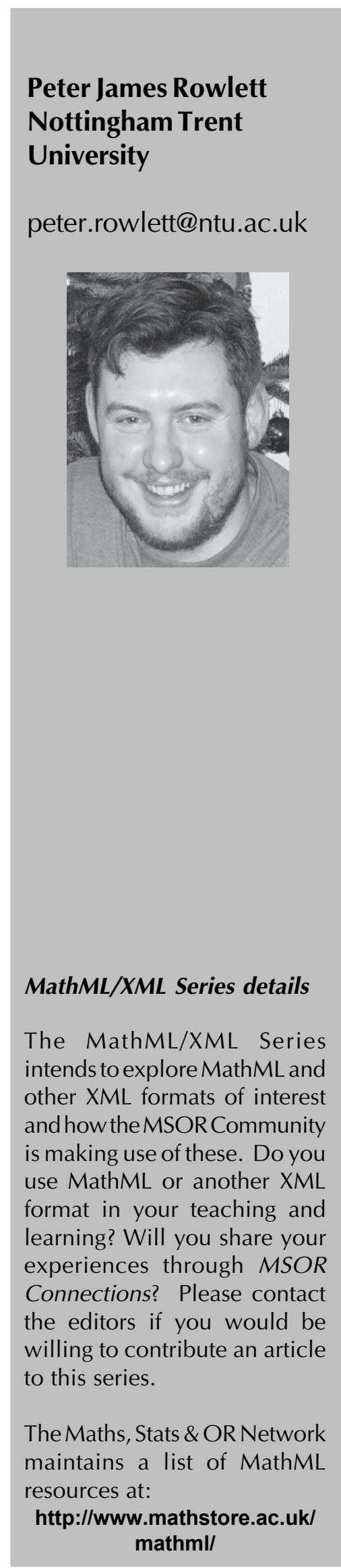

$\mathrm{Q}$ uite simply, MathML is a language for describing mathematics; this in itself is nothing special. A language such as LaTeX can describe mathematics, and converters exist to render this LaTeX as PDF or HTML but MathML is different, with MathML the meaning of the mathematics is encoded in a way that can be accessed by computer programs.

Automation is becoming increasingly important in the modern world. There are websites that will book trains, hotels and planes or ones that check stock levels, process credit card transactions and mark products for dispatch. The programs which run these tasks can only do so because the information needed is available in a machine-readable format.

With MathML, a program can be aware of the mathematical concepts behind the notation. A program that can interpret the mathematics and not simply process its notation is potentially capable of much greater levels of automation. This provides many obvious advantages and, as automation in other areas is revealing, potentially many that cannot currently be imagined.

Accessibility: Colleagues are finding increasingly that they have students on their courses who require materials in accessible formats - a large print format perhaps. Students are suggesting they will be best placed to access materials in a certain font and size. However, the materials being requested often only exist as hand written notes.

Typesetting these notes into LaTeX or another format means the source can be edited to fit the requirements of each student as they approach the lecturer. However, providing flexible delivery via MathML enables students to access the notes on the Web and adapt them to suit their requirements using web browser settings, or to use another program to convert the MathML e.g. into speech.

In terms of accessibility guidelines, the W3C Web Content Accessibility Guidelines require that "when an appropriate markup language exists, use markup rather than images to convey information... For example, use MathML to mark up mathematical equations" [1].

Materials produced as MathML will respond to the user's browser settings as well as the text on the page. The MathPlayer plugin [2] contains "an early version of Design Science's math-to-speech technology" and will interface with "most screen readers" through Microsoft's Active Accessibility interface [3].

Searchable mathematics: Writing computer programs with access to the meaning of the mathematics allows archives of mathematical materials to be produced that are searchable in a meaningful way; so particular pieces of mathematics might be searched for, along with the surrounding text. This can be useful on a basic level when finding a particular equation on a mathematics webpage, and on a more advanced level to write a MathML-capable search engine.

Computer-algebra systems:With MathML-encoded mathematics on a webpage, a user can copy and paste (or even drag and drop) an equation into a computeralgebra system, and then manipulate it. This provides the opportunity to quickly check a result on a web page, or to build on one. 
LaTeX and MathML: LaTeX is a typesetting language commonly used to create mathematical content. Wolfram Research [4] point out ambiguities that arise when computer systems try to analyse equations formatted only for typesetting and not semantic meaning, concluding that such languages are not suitable as input for computer mathematics systems. In fact, Wolfram Research regards as a "danger" averted the possibility that mathematical representation on the Web might be based on a typesetting language like TeX, stating that this "would have precluded, or made far more difficult, meaningful computational interaction with mathematical expressions found on the web."

Converters exist for MathML, and these can be used to convert, say, LaTeX code to MathML. It is important to bear in mind that the advantages of MathML as regards semantic information cannot necessarily be inferred, so that unless the source encodes the meaning of the mathematics, the conversion may not be able to do so accurately.

However, LaTeX conversion to MathML should be seen as an important step in MathML development. Firstly, much material already exists in LaTeX format; secondly, many users already know LaTeX and can start generating this as MathML where they might at the moment generate PDF.

Generating MathML: MathML is a more complex language than one like LaTeX, and much more verbose. So it is much more difficult to learn and to write. But MathML isn't intended to be written by a person typing the code. Instead, the mathematics is typed into a 'higher-level', simpler language or using a graphical input interface into a program that will encode it as MathML. In this sense, it is more like the PDF stage in publishing LaTeX code, rather than a replacement for the LaTeX stage.

Displaying MathML pages to older browsers: It is quite possible that a visitor to a webpage will not be able to render MathML code. By using a procedure called content negotiation, the web server can determine whether the web browser can accept MathML and if not provide an alternative rendering - perhaps by using a converter to a simpler format. Of course, such users would not be able to access many of the advantages of the MathML format until they obtain a MathML-enabled browser.

MathML as XML: Being an XML technology has advantages for MathML, being seen as a lot of work for quite a niche market, mathematics is not included in HTML. Languages such as LaTeX lacked widespread plug-in support for web browsers and were more commonly converted into inaccessible formats. As $\mathrm{XML}$ is much more widespread, MathML is able to utilise existing work in XML support to bring mathematics to the Web. The modular design of XML allows MathML to integrate effortlessly into other XML formats-allowing mathematics to be included in XHTML for webpages or SVG for graphics (diagrams, perhaps).

Using XSLT allows MathML (and all XML languages) to be transformed into other XML languages or to convert the content within MathML. Herman [5] suggests this can be used to "adapt to local conventions ('tan' vs ' $\operatorname{tg}$ ', ' $[a, b]^{\prime}$ vs ']a;b[', etc)".

Getting started with MathML: MathML on a webpage can be accessed already by those using a Mozilla browser, such as Firefox [6]. Those using Internet Explorer can download the free MathPlayer [2] plugin to access MathML content.

MathML can be converted to and from a number of formats, and there are programs that provide an interface to create MathML content. Interested readers should browse the W3C MathML Software List [7] and learn more about MathML from the $\mathrm{W} 3 \mathrm{C}$ resources page [8].

\section{References}

[1] Web Content Accessibility Guidelines. W3C, 1999. http://www.w3.org/TR/WAI-WEBCONTENT/ [Last accessed: 3 Jan 2006].

[2] MathPlayer: http://www.dessci.com/en/products/ mathplayer/ [Last accessed: 3 Jan 2006].

[3] MathPlayer Can Speak! [online]. Design Science, 2005. http://www.dessci.com/en/products/ mathplayer/tech/accessibility.htm [Last accessed: 3 Jan 2006].

[4] Wolfram Research. History of MathML [online]. Champaign, IL, USA: Wolfram Research, 2003. http://www.mathmlcentral.com/history.html [Last accessed: 3 Jan 2006].

[5] Herman, I. MathML [online]. W3C, 2005. Available at: http://www.w3.org/Consortium/ Offices/Presentations/MathML/ [Last accessed: 3 Jan 2006].

[6] Sidje, R.B., ed. MathML in Mozilla [online]. Mozilla.org, 2005. Available at: http:// www.mozilla.org/projects/mathml/ [Last accessed: 3 Jan 2006].

[7] Froumentin, M., ed. The W3C MathML software list [online]. W3C, 2005. http://www.w3.org/Math/ Software/ [Last accessed: 3 Jan 2006].

[8] W3C Math Home [online]. W3C, 2005. http:// www.w3.org/Math/ [Last accessed: 3 Jan 2006]. 